\title{
Justification of a synbiotic preparation ("probiotic+prebiotic") composition for use in veterinary practice
}

\author{
I.A. Gneusheva, N.E. Pavlovskaya, I.Yu. Solokhina*, and N.Yu. Ageeva \\ Oryol State Agrarian University, Generala Rodina st., 69, 302019 Oryol, Russia
}

\begin{abstract}
Studies have been carried out to substantiate the preparation's composition, which includes a complex of probiotic with prebiotic. It was found that the synbiotic basis is hydrolyzed lignin as a prebiotic with a concentration of $0.5-2.0 \%$, strain $L$. paracasei with bacterial cells concentration of $48 \mathrm{~B} \mathrm{CFU/g}$ with high probiotic activity and optimal biological properties, such as high adhesive activity, antagonistic activity against enteropathogenic microorganisms E. coli and S. aureus and acidforming activity. This preparation is recommended for use in veterinary practice to prevent and treat dysbiosis, increase the natural resistance of pigs' organism.
\end{abstract}

\section{Introduction}

Currently, livestock farms suffer significant losses because of the mortality of young farm animals due to infectious diseases of non-contagious etiology, which are associated with microbiocenosis violation of the gastrointestinal tract. The mortality rate of weaning piglets is up to $55 \%$ of total pig mortality. At the age of 15 days, most piglets die [1].

The development of environmentally friendly effective probiotic preparations that contribute to the intestinal microflora normalization of young farm animals, particularly pigs, is one of the ways to solve this problem [2].

To date, numerous studies prove the effectiveness of probiotic preparations use for the prevention and treatment of intestinal infections, dysbiosis in young agricultural animals [3].

Probiotics can be defined as "living microorganisms that benefit the health of the host when administered in sufficient quantities" [4]. Probiotics are characterized by a pronounced clinical effect in the treatment of acute intestinal infections; also, they do not violate the normal microflora biocenosis, increase immunity, stimulate and regulate digestion, greatly weaken stresses, provide high gains [5].

To date, the solution of the problem associated with the increase in the number of conditionally pathogenic microorganisms resistant to antibiotics remains promising. The problem is relevant in connection with the use of various lactobacilli strains in several probiotic preparations [6].

\footnotetext{
* Corresponding author: SolohinaIrina@yandex.ru
} 
In veterinary practice, bacteria of the Bacillus genus are often used in the composition of probiotics, which are alien microorganisms that have the following valuable properties: resistance to digestive system enzymes, being technological in production [7]. Since these bacteria are alien for intestinal microbiocenosis, they are quickly excreted from the gastrointestinal tract [8]. Organisms most used as probiotic agents are lactic acid bacteria Lactobacillus, which are normal microflora of the gastrointestinal tract [9].

Lactobacillus and similar bacteria, as well as their metabolites, can control pathogens such as Escherichia coli, Salmonella typhimurium and others [10].

Therefore, the development of a synbiotic preparation ("probiotic+prebiotic") based on homoprobiotic lactobacilli strains for veterinary practice is an actual task [11].

The purpose of this study was to assess the possibility of creating synbiotic "probiotic+prebiotic" preparation on the basis of homoprobiotic lactobacilli strains for use in veterinary medicine in order to prevent and treat dysbiosis and increase the natural resistance of farm animals.

\section{Objects of study}

Strains of lactobacilli used to conduct the study: L. paracasei, L. reuteri, L. salivarius isolated from the intestine contents of healthy weaning piglets; Lactobacillus acidophilus $L G-1-D E P-V G N K I$ isolated according to generally accepted methods from the "Lactobifadol" probiotic preparation (LLC BF "Component").

Enteropathogenic microorganisms: Escherichia coli and Staphylococcus aureus, obtained from intestinal contents of sick piglets were used to determine antagonistic activity of probiotic strains.

\section{Materials and methods}

The research used standard, original and generally accepted methods of microbiological and physico-chemical analysis.

Lactobacilli were grown at a temperature of $37^{\circ} \mathrm{C}$ for $1-2$ days in milk-serum media (MS).

Isolation of microorganisms from the large intestine contents of weaning piglets. Microorganisms were isolated from the piglets' intestinal contents, placed in sterile tubes with $0.9 \%$ solution of sodium chloride, the volume of which was $9 \mathrm{ml}$. Then, $1 \mathrm{~g}$ of stool masses were added to the test tubes with sterile wooden sticks with further suspending. The resulting suspension was settled for 5 minutes allowing for deposition of large particles. At the final stage, tenfold serial dilutions were prepared from the resulting suspension for subsequent inoculation by $0.1 \mathrm{ml}$ to selective nutrient media.

Microorganisms inoculation from a probiotic drug was carried out under aseptic conditions (laminar box with the 1st class of protection BAV — "Laminar-C" 105) to prevent infection with foreign microflora. To do this, milk agarized medium was prepared: $8 \mathrm{~g}$ of agar per $250 \mathrm{ml}$ of milk. It was sterilized and the preparation was applied with the help of a dispenser.

To determine the adhesive ability of bacterial cells, the methods of V.I. Brilis et al. (1986) and S. S. Gizatulina et al. (1991) were used. An express photocolorimetry method was used for the adhesive activity of lactobacilli. For lactobacilli adhesion, human red blood cells of 0 (I) blood groups with a positive rhesus factor were used as a substrate.

Determination of antagonistic activity of the studied lactobacilli strains was carried out on a petri dish on a standard nutrient medium by the growth zone delay magnitude.

The lactobacilli acid-forming activity was determined by the titrimetric method. 
Escherichia coli and Staphylococcus aureus were used to determine the antagonistic activity of strains by agar diffusion method.

Statistical results processing was carried out using MS Excel 2010. Studies of indicators were carried out in fivefold repetition. The obtained results are presented in the form of average values with confidence interval at $\mathrm{P}=0.95$.

\section{Results and Discussion}

Characteristics of basic biological properties of obtained lactobacilli Indicators of adhesive activity are one of the properties of probiotic microorganisms that stipulate the duration of their stay in the intestinal microbiome. Indicators of lactobacilli adhesion values in relation to human and pig erythrocytes are shown in table 1.

Table 1. Values of lactobacilli adhesion indicators in relation to human erythrocytes and pig erythrocytes $(\mathrm{x} \pm \mathrm{I} 95, \mathrm{n}=5)$

\begin{tabular}{|c|c|c|}
\hline \multirow{2}{*}{ Lactobacilli strains } & \multicolumn{2}{|c|}{ Adhesion rate, \% } \\
\cline { 2 - 3 } & human erythrocytes & pig erythrocytes \\
\hline $\begin{array}{c}\text { L. acidophilus } \\
\text { (control) }\end{array}$ & $45.8 \pm 7.5$ & $15.7 \pm 3.2$ \\
\hline L. paracasei & $46.4 \pm 0.5$ & $52.6 \pm 2.6$ \\
\hline L. salivarius & $6.4 \pm 1.3$ & $23.0 \pm 3.1$ \\
\hline L. reuteri & $10.6 \pm 4.8$ & $15.1 \pm 4.8$ \\
\hline
\end{tabular}

From the results of the obtained data it follows that the control strain L. acidophilus showed high adhesive properties in relation to human red blood cells; the strain exhibited low adhesive activity to pig erythrocytes. In relation to human red blood cells, L. salivarius exhibited low adhesive activity and showed an average level of adhesion to pig's erythrocytes. High adhesive activity was shown by bacteria strains $L$. paracasei against human red blood cells and pig erythrocytes.

The factor determining the probiotic lactobacilli potential is the ability to suppress the development of opportunistic microflora. The results of antagonistic lactobacilli activity in relation to enteropathogenic microflora obtained in sick animals from intestinal contents were obtained using agar diffusion method (Table 2).

Table 2. Indicators of antagonistic activity values of the studied lactobacilli strains $\left(x \pm I_{95}, n=5\right)$

\begin{tabular}{|c|c|c|c|c|}
\hline \multirow{2}{*}{$\begin{array}{c}\text { Strains } \\
\text { of conditionally } \\
\text { pathogenic } \\
\text { microorganisms }\end{array}$} & \multicolumn{4}{|c|}{ Diameter of growth delay zone, mm } \\
\cline { 2 - 5 } & L. paracasei & $\begin{array}{c}\text { L. } \\
\text { salivarius }\end{array}$ & L. reuteri & $\begin{array}{c}\text { L. acidophilus } \\
\text { (control) }\end{array}$ \\
\hline E. coli & $42.4 \pm 0.6$ & $32.8 \pm 1.4$ & $26.4 \pm 1.5$ & $42.3 \pm 3.1$ \\
\hline S. aureus & $32.4 \pm 3.4$ & $28.6 \pm 0.2$ & $24.6 \pm 2.4$ & $15.5 \pm 2.3$ \\
\hline
\end{tabular}

It was found that the tested lactobacilli strains can inhibit the growth and reproduction of certain enterobacteria, causing the manifestation of diarrheal diseases in piglets.

The optimal value of the antagonistic activity indicator compared to control was revealed in the L. paracasei strain. For this strain, the diameter of the growth delay zone in relation to E.coli was $42.4 \mathrm{~mm}$, and to $S$. aureus $-32.4 \mathrm{~mm}$.

Antagonistic lactobacilli activity is associated with indicators of acid formation. The results of the obtained values of probiotic strains' limiting acid formation are presented in table 3 . 
Table 3. Indicators of acid-forming activity of probiotic lactobacilli strains $\left(x \pm I_{95}, n=5\right)$

\begin{tabular}{|c|c|}
\hline Probiotic strain under study & Indicators of limit acid formation, $\mathrm{T}^{\mathrm{o}}$ \\
\hline L. acidophilus (control) & $406 \pm 5.2$ \\
\hline L.paracasei & $420 \pm 6.4$ \\
\hline L.salivarius & $382 \pm 12.2$ \\
\hline L. reuteri & $334 \pm 8.4$ \\
\hline
\end{tabular}

From the obtained data it follows that the optimal indicator value of limit acid formation was revealed in the control strain L. acidophilus and studied L. paracasei, , which the limit acid formation index value was $420 \mathrm{~T}^{\circ}$, which is 14 degrees higher than the reference value.

Consequently, during studies of indicators that determine the main biological properties of lactobacilli strains, L. paracasei, was identified as the most promising strain showing high adhesive properties in relation to human erythrocytes $(56.5 \%)$ and pig $(50.8 \%)$, possesses antagonistic activity against enteropathogenic microorganisms and the optimal values of acid formation $\left(420 \mathrm{~T}^{\circ}\right)$.

In the laboratory sample development of a synbiotic ("probiotic+prebiotic") preparation based on the selected strain for the cultivation of the L.paracasei strain, the MRS medium with cabbage decoction was used.

The strain cultivation was carried out by the depth method in the Biostat A plus bioreactor with 3 liters volume at $\mathrm{t}^{\mathrm{0}} 37^{\circ} \mathrm{C}, \mathrm{pH}-6.2-8.0$, agitator rotation speed $-160 \mathrm{rpm}$, aeration - 4 liters per minute.

L.paracasei with a final concentration of $10 \%$ was used as an inoculation culture. Inoculation culture was introduced to 2.5 liters of nutrient medium in the amount of $100 \mathrm{ml}$. Cultivation was carried out for 24 hours. The $\mathrm{pH}$ of the medium was not adjusted. The growth of lactobacilli concentration is shown in Figure 1.

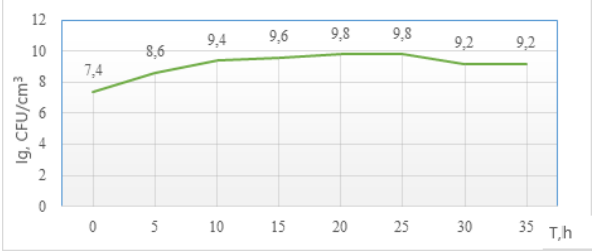

Fig. 1. Dynamics of L.paracasei concentration growth indicators in culture from cultivation time

The indicator of the final biological concentration of lactobacilli amounted to $6.0 \cdot 10^{9}$ $\mathrm{CFU} / \mathrm{cm}^{3}$ in native culture.

At the next stage, a liquid form of the preparation based on native culture was obtained; its characteristics are presented in table 4.

Table 4. Main characteristics' indicators of native culture L. paracasei

\begin{tabular}{|c|c|}
\hline Indicator & Indicator value \\
\hline General characteristics & Native culture \\
\hline Biological concentration, $\mathrm{CFU} / \mathrm{cm}^{3}(\mathrm{~g})$ & $6.0 \cdot 10^{9}$ \\
\hline $\mathrm{pH}$, units & $3.6 \pm 0.1$ \\
\hline
\end{tabular}

Based on $L$. paracasei strain, a synbiotic preparation's laboratory sample in dry lyophilized form with bacterial cells concentration of $48 \mathrm{~B} \mathrm{CFU/g}$ on hydrolyzed lignin (GOST R 8.563-96) was obtained.

Pharmacological effect of synbiotic preparation. The resulting preparation is a preparation of microbial origin based on hydrolysed lignin, which adsorbs products of microbial origin, including toxins of various nature, heavy metals, radioactive isotopes, 
ammonia, allergens, xenobiotics and contributes to their excretion through the gastrointestinal tract.

This preparation has an action against diarrhea, is an antioxidant, enterosorbent, exhibits detoxifying effect positively affecting the general microbiota of the large intestine, enriches human food with natural dietary fibers.

Pharmacokinetics of the synbiotic preparation: excreted through the intestine in an unchanged form.

The obtained samples were used in the experiment of assessing its effect on the species and quantitative composition of intestinal microflora.

Since the E. coli and $S$. aureus strains are characterized by multiple antibiotic resistance, the actual issue was the study of the probiotic preparations' influence on their survival.

The results of the studied probiotic preparations' influence in relation to clinical $E$. coli and $S$. aureus isolates are presented in Table 5.

Table 5. Influence of studied probiotic preparations on the survival of clinical E. coli and S. aureus isolates $(n=10)$.

\begin{tabular}{|c|c|c|c|c|c|}
\hline \multirow{2}{*}{$\begin{array}{c}\text { Clinical } \\
\text { isolates }\end{array}$} & $\begin{array}{c}\text { Control } \\
\text { probiotic } \\
\text { preparation }\end{array}$ & 0.25 & 0.5 & 1.0 & 2.0 \\
\cline { 2 - 6 } & \multicolumn{6}{|c|}{ Concentration of prebiotic in complex, \% } \\
\cline { 2 - 6 } & 668 & 1.4 & - & - & - \\
\hline E. coli & 616 & 1.7 & - & - & - \\
\hline S. aureus & &
\end{tabular}

It was found that a synbiotic preparation with a concentration of hydrolyzed lignin lying in the range of $0.5 \%$ to $2.0 \%$ suppresses the growth of pathogenic bacteria. The preparation with $0.25 \%$ lignin concentration showed a decrease in antibacterial activity against E. coli and $S$. aureus isolates.

The obtained results indicate that the synbiotic preparation based on L.paracasei in the hydrolyzed lignin complex as a prebiotic has a high probiotic activity against E. coli and $S$. aureus clinical isolates.

Studies have shown that probiotic preparations showed antagonistic activity against test cultures. The "Lactobifidol" preparation was used as control, which is less effective in comparison with a complex synbiotic preparation based on the L.paracasei strain. The results of antibacterial activity of the studied probiotic preparations are presented in tables $6,7$.

Table 6. Antibacterial activity of probiotic preparations in relation to E. coli (agar diffusion method)

\begin{tabular}{|c|c|c|c|}
\hline \multirow{2}{*}{$\begin{array}{c}\text { Producer and } \\
\text { Component Name }\end{array}$} & \multicolumn{2}{|c|}{ Growth delay zone, $\mathrm{mm}$} & Variation coefficient, \% \\
\cline { 2 - 4 } & $\lim$ & $\mathrm{x} \pm \sigma$ & $\mathrm{V}$ \\
\hline $\begin{array}{c}\text { Complex preparation } \\
\text { ("probiotic+prebiotic") }\end{array}$ & $24-31$ & 26.7 & 32.9 \\
\hline $\begin{array}{c}\text { "Lactobifadol" } \\
\text { (control) }\end{array}$ & $19-24$ & 20.6 & 21.2 \\
\hline
\end{tabular}

Table 7. Antibacterial activity of probiotic preparations in relation to S. aureus (diffusion method)

\begin{tabular}{|c|c|c|c|}
\hline Producer and Component & \multicolumn{2}{|c|}{ Growth delay zone, $\mathrm{mm}$} & Variation coefficient, \% \\
\cline { 2 - 4 } Name & $\lim$ & $\mathrm{x} \pm \sigma$ & $\mathrm{V}$ \\
\hline $\begin{array}{c}\text { Complex preparation } \\
\text { ("probiotic+prebiotic") }\end{array}$ & $24-31$ & 29.1 & 31.4 \\
\hline $\begin{array}{c}\text { "Lactobifadol" } \\
\text { (control) }\end{array}$ & $19-24$ & 19.4 & 19.4 \\
\hline
\end{tabular}


It was found that the synbiotic preparation based on L. paracasei strain with bacterial cells concentration of $48 \mathrm{~B} \mathrm{CFU} / \mathrm{g}$ and with a concentration of hydrolyzed lignin from 0.5 $2.0 \%$ has antagonistic activity against opportunistic microorganisms $(22-31 \mathrm{~mm})$. Further research should be directed to the creation of the hardware and technological production line of a new synbiotic preparation based on L. paracasei homoprobiotic strain to be used in veterinary practice for the prevention and treatment of dysbiosis and increase of the natural farm animals bodies' resistance.

\section{Conclusions}

Thus, the prospects of using homoprobiotic microorganisms in the creation of microbiocenosis correction means of farm animals are confirmed by the obtained results of the study. The composition of the synbiotic "probiotic+prebiotic" preparation for use in veterinary practice was substantiated.

To sum up, it can be concluded that an era of successful use of probiotics and prebiotics in clinic for the gastrointestinal tract diseases treatment has started. It is proved that the use of probiotics is effective in certain diseases. However, there are unresolved remaining issues related to screening specific strains for farm animals of each species, the selection of optimal therapeutic doses needed to achieve sustained remission. As a consequence of the individual characteristics of the intestinal microbiome, it is likely that new clinical studies will answer questions about the safety and effectiveness of pro- and prebiotics.

The prospect of further research is certainly related to the information already available in this area.

\section{References}

1. I.N. Tokarev, A.V. Bliznetsov, S.R. Ganieva, Scientific notes of the Kazan state academy of veterinary medicine named after N.E. Bauman, 219(3), 275 (2014)

2. V. Stepanova, Effective animal husbandry, 8(165), 22 (2020)

3. V. Bondarenko, Pharmateka, 2(196), 26 (2010)

4. Y. Wang, Y. Wu, B. Wang et al. AMB Expr., 7, 52 (2017)

5. N.I. Ursova, Pediatric pharmacology, 2(10), 46 (2013)

6. E. A. Moskalenko, N. N. Zabashta, Collection of scientific works of the North Caucasian scientific research institute of livestock, 3(5), 150 (2016)

7. A.V. Savustyanenko, Actual infectology, 2(11), 35 (2016)

8. M.K. Koilybayeva, G.O. Ustenova, D. Batyrbayeva, Zh.S. Alibayeva, K.K. Bulletin of Kaz NMU, 4, 181 (2018)

9. C. Hou, X. Zeng, F. Yang et al, J. Animal Sci Biotechnol, 6, 14 (2015)

10. C. Suo, Y. Yin, X. Wang et al, BMC Vet Res 8, 89 (2012)

11. E.E. Shkolnikov, N.K. Eremets, I.V. Pavlenko, L.A. Neminuschaya, T.A. Skotnikova, E.F. Tokarik, I.V. Bobrovskaya, D.N. Filimonov, V.V. Gavrilov, I.V. Kovalskiy, et al, Bulletin of Kazan Technological University, 13, 255 (2014) 